\title{
Comprehensive transcriptome analysis of reference genes for fruit development of Euscaphis konishii
}

\author{
Cheng-Long Yang Equal first author, ${ }^{1}$, Xue-Yan Yuan ${ }^{\text {Equal first author, } 2,3}$, Jie Zhang ${ }^{1}$, Wei-Hong Sun ${ }^{2,3}$, Zhong-Jian Liu ${ }^{2,3}$, Shuang- \\ Quan Zou Corresp. 2, 3 \\ ${ }^{1}$ Biotechnology Institute, Fujian Academy of Agricultural Sciences, Fuzhou, Fujian, China \\ 2 Fujian Colleges and Universities Engineering Research Institute of Conservation and Utilization of Natural Bioresources at College of Forestry, Fujian \\ Agriculture and Forestry University, Fujian, Fuzhou, China \\ ${ }^{3}$ Key Laboratory of National Forestry and Grassland Administration for Orchid Conservation and Utilization at College of Landscape Architecture, Fujian \\ Agriculture and Forestry University, Fuzhou, Fujian, China \\ Corresponding Author: Shuang-Quan Zou \\ Email address: zou@fafu.edu.cn
}

Background: Quantitativereal-time reverse transcriptase polymerase chain reaction is the common method to quantify relative gene expression. Normalizating using reliable genes is critical in correctly interpreting expression data from qRT-PCR. Euscaphiskonishii is a medicinal plant with a long history in China, which has various chemical compounds in fruit. However, there is no report describing the selection of reference genes in fruit development of Euscaphiskonishii.

Methods: We selected eight candidate reference genes based on RNA-seq database analysis, and ranked expression stability using statistical algorithms GeNorm, NormFinder, BestKeeper and ReFinder. Finally, The nine genes related to the anthocyanin synthesis pathway of Euscaphis konishii were used to verify the suitability of reference gene.

Results: The results showed that the stability of EkUBC23, EkCYP38 and EkGAPDH2 was better, and the low expression reference genes (EKUBC23 and EKCYP38) were favourable for quantifying low expression target genes, while the high expression reference gene (EKGAPDH2) was beneficial for quantifying high expression genes. In this study, we present the suitable reference genes for fruit development of Euscaphis konishii based on transcriptome data, our study will contribute to further studies in molecular biology and gene function on Euscaphis konishii and other closely related species. 
4 Comprehensive Transcriptome Analysis of Reference

5 Genes for Fruit Development of Euscaphis konishii

Cheng-Long Yang ${ }^{1}$, Xue-Yan Yuan ${ }^{2,3}$, Jie Zhang1, Wei-Hong Sun ${ }^{2,3}$, Zhong-Jian Liu ${ }^{2,3}$, Shuang-Quan Zou 2,3,*

${ }^{1}$ Biotechnology Institute, Fujian Academy of Agricultural Sciences, Fuzhou,Fujian, China ${ }^{2}$ Fujian Colleges and Universities Engineering Research Institute of Conservation and Utilization of Natural Bioresources at College of Forestry, Fujian Agriculture and Forestry University, Fuzhou, Fujian, China

${ }^{3}$ Key Laboratory of National Forestry and Grassland Administration for Orchid Conservation and Utilization at College of Landscape Architecture, Fujian Agriculture and Forestry University, Fuzhou, Fujian, China

Corresponding Author:

Shuang-Quan Zou $2,3, *$

Street Address, Fuzhou, Fujian, 350002, China

Email address: zou@fafu.edu.cn

\section{Abstract}

Background: Quantitative real-time reverse transcriptase polymerase chain reaction is the common method to quantify relative gene expression. Normalizating using reliable genes is critical in correctly interpreting expression data from qRT-PCR. Euscaphis konishii is a medicinal plant with a long history in China, which has various chemical compounds in fruit. However, there is no report describing the selection of reference genes in fruit development of Euscaphis konishii.

Methods: We selected eight candidate reference genes based on RNA-seq database analysis, and ranked expression stability using statistical algorithms GeNorm, NormFinder, BestKeeper and ReFinder. Finally, The nine genes related to the anthocyanin synthesis pathway of Euscaphis konishii were used to verify the suitability of reference gene.

Results: The results showed that the stability of $E k U B C 23, E k C Y P 38$ and $E k G A P D H 2$ was better, and the low expression reference genes ( $E k U B C 23$ and $E k C Y P 38)$ were favourable for 
37 quantifying low expression target genes, while the high expression reference gene (EkGAPDH2)

38

39

40

41

42

43

44

45

46

47

48

49

50

51

52

53

54

55

56

57

58

59

60

61

62

63

64

65

66

67

68

69

70

71

72

73

74

75

76

was beneficial for quantifying high expression genes. In this study, we present the suitable reference genes for fruit development of Euscaphis konishii based on transcriptome data, our study will contribute to further studies in molecular biology and gene function on Euscaphis konishii and other closely related species.

\section{Introduction}

As an important economic crop, fruit trees play an important role in the agricultural production of various countries. Fruit development research is an important part of fruit science. Screening out stable reference genes in the fruit developmental stage can lay the foundation for the study of functional genes in neighbouring species and accelerate the process of molecular breeding.

The analysis of gene expression, or more correctly tanscript abundance, is essential in all aspects of molecular biology research to understand gene expression patterns in different biological processes (Dussert et al., 2013). So far, the commonly used methods for the detection of gene expression are northern blotting, gene chip and quantitative real-time reverse transcription polymerase chain reaction (qRT-PCR). Among them, qRT-PCR has become one of the most powerful tools for studying gene expression due to its high sensitivity, accuracy and specificity (Bustin SA, 2002; Nolan, Hands \& Bustin, 2006). qRT-PCR is wonderful for fast and accurate gene expression analysis. However, this technology requires suitable reference genes to normalize expression data and control the quantity of cDNA (Derveaux S, Vandesompele J \& Hellemans J, 2010). In previous stages, housekeeping genes were selected as stable reference genes. However, lots of housekeeping genes have significantly different levels of expression in different experimental conditions or related species (Dheda K et al., 2004). The stability of reference gene is influenced by experimental conditions and plant species. It is necessary to find additional reference genes for most plants.Therefore, according to different experimental conditions, the key is to accurately quantify target genes and screen one or more reference genes with stable expression from multiple candidate reference genes.

Currently, Gene Expression Omnibus was used as a new tool to screen reference genes due to its accuracy and comprehensiveness. GeneChip research of Arabidopsis thaliana revealed that the protein phosphatase $2 \mathrm{~A}$ was favourable for quantifying low expression target genes (Czechowski T et al., 2005); Coker and Davies' research showed that the stability of TUA (Tubulin alpha), CYP (Cyclophilin) and GAPDH (Glyceraldehyde-3-phosphate dehydrogenase) is better by using dbEST (Coker J S \& Davies E, 2004). The best reference genes of Elaeis guineensis (Xia W et al., 2014), Populus trichocarpa (Pang QQ et al., 2017) and Populus trichocarpa (Su XJ et al., 2013) were screened out based on RNA-seq technology.

Euscaphis konishii is a member of the family Staphyleaceae, which is used as an ornamental and medicinal source material in China. The fruit of E. konishii has various chemical compounds, including flavonoid compounds (Liang WX et al., 2018a), triterpene compounds (Cheng JJ et al.; Li YC et al.,2016), and phenolic acid compounds (Huang Y et al., 2014), which have both anti-inflammatory and anticancer effects according to traditional and modern medical

Peer) reviewing PDF | (2019:05:37531:4:0:NEW 6 Dec 2019) 
77 research. Moreover, in order to study the gene regulation mechanisms of medicinal compounds

78 in E. konishii, several reference genes in E. konishii were selected by Liang (Liang WX et al., 79 2019) based on transcriptome data (Liang WX et al., 2018b). However, the data being studied 80 from Liang et al. lack the transcriptome of the fruit at different developmental stages. Yuan 81 identified a large number of differentially expressed genes associated with endocarp colouration 82 based on the transcriptome database (Yuan WY et al., 2018), but it is unknown which genes are 83 suitable as reference genes related to fruit development. Huang revealed that the total triterpenes 84 of Euscaphis konishii pericarp showed the immune boosting effect and hepatic protective activity 85 against inflammation, oxidative stress and apoptosis in BCG/LPS-induced liver injury. These 86 findings suggest that pericarp of $E$. konishii might be a promising natural food for 87 immunological hepatic injury (Huang $\mathrm{W}$ et al., 2019). Because of the information on gene 88 expression in E. konishii is still unclear, transcriptome technology would play an important role 89 in mining the reference gene. Our study provided two available internal control genes for qRT90 PCR data normolizetion in E. konishii. It will contribute to analyze the expression patterns of 91 compound related gene, and further to reveal the breeding mechanism.

\section{Materials \& Methods}

93 Plant material

94 The fruits of E. konishii have three developmental stages, including the green stage, turning stage, and red stage. These were collected from Qingliu County, Fujian Province, China, from June to September. The samples were provided by Sanming yisheng agricultural and forestry co., LTD. All pericarp samples were separated from the fruit, wrapped in tin foil and then frozen in liquid nitrogen and immediately stored at $-80^{\circ} \mathrm{C}$ until they were qualified for further analysis. Three biological replicates for each sample were used for RNA extraction.

\section{Establishment of the RNA-Seq database}

We sequenced the transcriptome of the E. konishii pericarp in three developmental stages. The library produced $67.78 \mathrm{G}$ of clean data, and the average clean data in the nine samples were 6.78 G. In this study, high-quality libraries with mapping rate were higher than $79.74 \%$ and Q30 values highter than $92.70 \%$. In total, 86,120 unigenes with a mean length of $893.34 \mathrm{bp}$ and N50 length of 1,642 nt were assembled by using Trinity (Grabherr et al, 2011) with min_kmer_cov set to 2 by default and all other parameters set default. The length distribution of all unigenes were shown in Figure 1. The raw data has been submitted to NCBI, Sequence Read Archive (SRA) submission: SRR9267648 to SRR9267656. We used BLAST software to compare unigene sequences to NR (ftp://ftp.ncbi.gov/blast/db/), Pfam (http://pfam.xfam.org/), GO (http://www.geneontology.org/), KEGG(http://genome.jp/kegg/), Pwiss-Prot (http://uniprot.org/), 
112 (ftp://ftp.ncbi.nih.gov/pub/COG/). Transcriptome sequences were used as references to analyse

113 the expression profiles of each sample. The sequencing results of each sample were compared to

114 the reference sequence, and the expression amount of each unigene in different samples was

115 obtained.

116 RNA-Seq database analysis and primer design

117 Genes with expression levels lower than 5 FPKM in tanscriptomes would make poor qPCR

118 references due to the difficulties in detecting and quantifying their expression. (Kimmy AS,

119 Patrick PE\& Joshua RP, 2017). After their removal, a total of 75,048 genes in E. konishii were

120 evaluated. We calculated the mean expression value, standard deviation, and coefficients of

121 variation based on the raw RNA-seq data. Base on the requirements $\mathrm{CV} \leq 0.2$ and $\mathrm{FC} \leq 0.2$,

122 1,131 genes (Table S1) were obtained by removing overabundant genes with low expression

123 levels. Finally, we selected the eight candidate genes according to genome annotation of each,

124 which was assigned base on the best mach of the alignments using Blast to NR, TrEMBL and

125 KEGG databases. The selecting steps of candidate reference genes were shown in Fig S1. In

126 order to ensure the accuracy of the reference gene predictions, the coding sequences of eight

127 selected genes was used as queries for BLAST orderly through the TAIR database

128 (http://www.arabidopsis.org/). The sequences with the highest homology with Arabidopsis were 129 shown in Table 1.

130 Here we obtained the nucleotide sequences of reference genes from our laboratory. The 131 nucleotide sequences of candidatate reference genes were appended in Table S2. According to 132 the nucleotide sequence of candidate reference genes and the design principle of qRT-PCR, 133 forward and reverse primers of all candidate reference genes were designed using Primer

134 Premier 6.0 software and were synthesized by Jinweizhi Biotechnology Co., LTD (Suzhou, 135 China) and purified by PAGE. The primer sequences, amplicon size, anneal temperature were 136 shown in Table 2.

137 RNA Extraction and cDNA Synthesis.

138 Total RNA was extracted and purified by using an RNAprep Pure Plant Kit (Polysaccharides 139 and Polyphenolics-Rich, Tiangen, Beijing, China), according to the manufacturer's instructions.

140 Two-hundred nanograms of total RNA of each sample was used as the template. In addition, the 141 cDNA synthesis strand was performed by using a Revert Aid First Strand cDNA Synthesis Kit

142 (Thermo Fisher, Foster City, CA, USA), according to the manufacturer's instructions and was 143 stored at $-80^{\circ} \mathrm{C}$ for further experiment. 
144 Candidate reference genes for RT-PCR amplification

145 The RT-PCR mixture contained $25 \mu \mathrm{L}$ of $2 \times$ EasyTaq ${ }^{\circledR P C R}$ SuperMix, $0.4 \mu \mathrm{L}$ of forward $146 \operatorname{primer}(10 \mu \mathrm{M}), 0.4 \mu \mathrm{L}$ of reverse primer $(10 \mu \mathrm{M})$, and $5 \mu \mathrm{L}$ of diluted cDNA in a final volume 147 of $50 \mu \mathrm{L}$. PCR conditions: 40 cycles of $3 \mathrm{~min}$ at $94^{\circ} \mathrm{C}, 94^{\circ} \mathrm{C}$ at $30 \mathrm{~s}, 56^{\circ} \mathrm{C}$ at $10 \mathrm{~s}, 72^{\circ} \mathrm{C}$ at $30 \mathrm{~s}$, 148 and $72^{\circ} \mathrm{C}$ at 7 min. RNA quality was determined by $1.2 \%$ agarose gel electrophoresis.

149 Candidate reference genes for qRT-PCR amplification

150 The qRT-PCR mixture contained $3 \mu \mathrm{L}$ of diluted cDNA, $5 \mu \mathrm{L}$ of $2 \times$ SYBR Green Master, 0.4 $151 \mu \mathrm{L}$ of forward primer $(10 \mu \mathrm{M})$, and $0.4 \mu \mathrm{L}$ of reverse primer $(10 \mu \mathrm{M})$, with $\mathrm{ddH}_{2} \mathrm{O}$ added to 152 achieve a final volume of $10 \mu \mathrm{L}$. All PCRs were performed using the QuantStudioTM Real-Time 153 PCR System under the following conditions: 40 cycles of $2 \mathrm{~min}$ at $95^{\circ} \mathrm{C}, 5 \mathrm{~s}$ at $95^{\circ} \mathrm{C}$ and $30 \mathrm{~s}$ at $15460^{\circ} \mathrm{C}$. The procedure ended with a melt-curve ramping from $60-95^{\circ} \mathrm{C}$. The melting curve was 155 analysed to determine primer specificity. For further confirmation, PCR products were cloned 156 into pGXT vectors respectively and then sequenced by Biosune Biotech (Fuzhou, China).

\section{Statistical analysis}

158 The instrument calculated the Cq value of each sample based on the qRT-PCR experiment. 159 We can analyse the stability of candidate reference genes using GeNorm, NormFinder, and 160 BestKeeper software. Finally, ReFinder was used to calculate the comprehensive ranking of the 161 stability of candidate reference genes based on analysis results.

Anthocyanin is the key factor affecting fruit colouration as an important plant pigment. In general, fruit colouration is closely related to the content and proportion of anthocyanin. To verify the results of our experiments, the expressions of nine genes related to the anthocyanin synthesis pathway were calculated with the reference genes selected (Fig. S5). Moreover, the EkSS3 gene related to glycometabolism pathway was also used to validate the reliability of reference genes. The details of primer were shown in Table 3. The qRT-PCR experimental method was the same as described above, and the relative expression level was calculated by the $2^{-\Delta \Delta c t}$ method. Experimental data from three biological replicates were analysed using analysis of variance (ANOVA), followed by Student's t-test $(\mathrm{P}<0.05)$. 
175 Based on the theree comparisons of green vs. turning, green vs. red, and turning vs. red, we 176 identified a total of 4804 differentially expressed genes (DEGs). Among them, 2175 DEGs

177 between green vs. turning, 3935 DEGs between green vs. red, and 936 DEGs between turning vs. 178 red were detected. Among the 4804 DEGs selected to predict functions by gene ontology (GO) 179 annotation and KEGG pathway analysis. The genes related to the glycometabolism, anthocyanin 180 biosynthesis, flavonoid biosynthesis and carotennoid biosynthesis performed different expression 181 in different stages of fruit ripening.

\section{Reference gene selection and primer design}

183 According to selecting process (Fig. S1), eight candidate reference genes were chosen to 184 perform the gene normalization studies. The sequences of these candidate reference gene were 185 used to design the qRT-PCR primers. The details of eight reference genes were lised in Table 1. 186 The gene models of eight candidate reference refered to the homologous genes of Arabidopsis, 187 and the details were shown in Figure S2.

188 Candidate reference genes for RT-PCR amplification

189 PCR results of the 8 candidate internal reference genes are shown in Figure S4 with a single 190 band, no primer dimer and nonspecific amplification, which could be used for subsequent qRT191 PCR analysis. We sequenced the PCR amplification products of eight genes and blast the 192 correctness of the PCR amplified fragments (Table S3).

193 Cq values of candidate reference genes

194 The Cq values for all 8 reference genes are shown in Figure 2. The Cq values varied from 19524.65 (EkGAPDH2) to 35.27 (EkACT7). Moreover, as shown in Figure 2, EkmMDH2, EkMDH 196 and $E k A C T 7$ are more variable than other candidate reference genes. The stability of $E k m M D H 2$, $197 E k M D H$ and $E k A C T 7$ expression is poor.

198 qRT-PCR analysis

199 As shown in Figure S3, the melting curve of the 8 candidate reference genes at different 200 developmental stages only had a single main peak, and the amplification curves between the 201 repeated samples had high repeatability.

202

\section{Expression stability analysis of reference genes}

203 Evaluating the expression stability of candidate reference genes depended on statistical 204 analysis. Four different statistical software programs (GeNorm, NormFinder, BestKeeper and 205 ReFinder) were commonly used to calculate the variability of the candidate genes expression 206 and determine the one which were the most suitable. 
207

208

209

210

211

212

213

214

215

216

217

218

219

220

221

222

223

224

225

226

227

228

229

230

231

232

233

234

235

236

237

Gene expression stability was determined by the M-value in GeNorm. The gene expression stability increases as the M-value decreases (Wu JY et al., 2018; Vandesompele J et al., 2002). It was considered that candidate reference genes can be used as reference genes when the M-value is less than 1.0; the result of GeNorm revealed that $E k U B Q 1$ was the most stable gene with the lowest M-value, followed by EkUBC23, EkCYP38 and EkGAPDH2. However, EkACT7,

$E k m M D H 2, E K M D H$ and $E k T U A 3$ were unsuitable as reference genes, with a value greater than 1.0. Expression stability values analysed by GeNorm are shown in Figure 3-A.

NormFinder software ranks all reference gene candidates based on intra- and inter-group variation and combines results into a stability value for each candidate reference gene. A better stable gene expression is indicated by a lower stability value (Andersen CL et al., 2004). The analysis results of NormFinder shown in Figure 3-B revealed that EkGAPDH2 was the most stable gene for all samples.

BestKeeper software determines the most stably expressed genes based on the standard deviation (SD). The lower the SD, the greater the reference gene expression stability will be (Pfaffl MW et al., 2004). As the analysis results in Figure 3-C show, the SD values of EkACT7, $E k m M D H 2, E k M D H, E k T U A 3$ and $E k G A P D H 2$ are greater than 1 and are considered unacceptable as reference genes, according to the selection criteria of BestKeeper software. $E k U B C 23, E k U B Q 1$ and $E k C Y P 38$ are stable genes with low SD values.

ReFinder software was used to rank the stability obtained by the analysis of GeNorm, NormFinder and BestKeeper on the comprehensive index. The stability of the reference gene expression is directly related to the size of the index. According to the analysis result of ReFinder shown in Figure 3-D, the stability ranking obtained by ReFinder software is as follows: EkUBC23, EkCYP38, EkGAPDH2, EkUBQ1, EkMDH, EkTUA3, EkmMDH, and EkACT7. The results showed that the stability of $E k U B C 23, E k C Y P 38$ and $E k G A P D H 2$ was better, and the low expression reference genes $(E k U B C 23$ and $E k C Y P 38)$ were favourable for quantifying their targets, while the high expression reference gene $(E k G A P D H 2)$ was beneficial for quantifying its target.

\section{The suitability of the reference gene}

According to the results of the four algorithms (GeNorm, NormFinder, BestKeeper and ReFinder), EkUBC23, EkCYP38 and EkGAPDH2 performed more stably. EkUBC23 and EkCYP38 showed similar expression levels during fruit developmental stages. However, 
$238 E k U B C 23$ was used to normalize the expression due to its greater stability. Relative expression

239 levels were normalized using the low expression reference gene (EkUBC23) and high expression 240 reference gene $(E k G A P D H 2)$. The results showed that there were some differences between the 241 expression levels of 9 genes related to the anthocyanin synthesis pathway and transcriptome 242 sequencing (Figure 4). When $E k U B C 23$ was used as the reference gene, the results had no 243 significant difference. When $E k G A P D H 2$ was used for normalizing low expression target genes 244 [c57877.graph_c0(DFR),c59825.graph_c0(CHS) and c69862.graph_cl(UFGT)], the results 245 were different from the expression of transcriptome. However, EkGAPDH2 has better accuracy 246 than $E k U B C 23$ when it was used for normalizing high expression target genes 247 [c72659.graph_c0(CHS) and c72737.graph_c0(CHI)]. Therefore, we suggest that the stability 248 and expression of reference genes should be considered as important selection conditions.

249 To ensure the reliabilty of result, We chosen EkSS3 gene related to glycometabolism pathway 250 as target gene. Relative expression levels were normalized using two most stable reference genes 251 (EkGAPDH2 and EkUBC23). As shown in Figure S6, when normalized using EkGAPDH2 and $252 E k U B C 23$ as reference gene respectively, the results had no significant difference. Compared 253 with the green stage, EkSS3 expression was clearly downregulated at turning stage and red stage. 254 The EkGAPDH2 and EkUBC23 performed well when normalizing target gene in different 255 pathway.

256

257

258

\section{Discussion}

Many studies have shown that it was quicker and more efficient to screen appropriate reference genes based on transcriptome data. In addition, primers designed according to the 260 transcriptome sequencing of the material itself are more stable than those using other materials. Selection of the reference gene based on transcriptome data has been done in many plant species, such as Corylus (Yang D et al., 2017), Sedum alfredii (Sang J et al., 2013) and Dendrocalamus 263 sinicus [Guo XJ et al., 2018], and so on. In the present study, we selected 3 stable reference 264 genes (EkUBC23, EkCYP38 and EkGAPDH2) based on transcriptome data of E. konishii pericarp in three different developmental stages. This result validates and complements the 266 results of reference gene screening by Liang. (Liang WX et al., 2018b). external environmental conditions. $A C T$ was commonly used as a reference gene. For example, it 
269 was the most stable gene in studies of Glycine max (Ma SH et al., 2013). In contrast, we confirm 270 that $A C T$ is the most unstable gene in our study. This consequence is consistent with studies in 271 Setaria viridis (Martins PK et al., 2017) and E. konishii by Liang et al. (Liang WX et al., 2018b).

$272 U B C$ has been widely accepted for normalization of gene expression as a reference gene (Yuan 273 W et al., 2012). UBC was selected as the reference gene in the study of Prunus pseudocerasus (2 274 Zhu YY et al., 2015). However, it was unsuitable as a reference gene for Oryza sativa (Li QF et $275 a l ., 2008)$. In this study, we identified $U B C$ as a reference gene to quantify low expression target 276 genes. CYP played important roles in Elaeis guineensis(Yeap WC et al., 2014) and Malus 277 domestica (Kumar G \& Singh A K, 2015) as reference a gene. CYP with stability and low 278 expression is also used for quantifying low expression target genes. GAPDH, an enzyme in 279 glycolysis, has been widely used as a reference gene in different species (Kozera B\& Rapacz M, 280 2013). However, GAPDH has different performances in different species and different 281 experimental conditions. GAPDH shows stable expression in Citrus sinensis (Wu JX et al., 282 2014)and Lycopersicon esculentum(Mascia T et al.,2010), but it did not perform well in $S$. 283 viridis(Martins PK et al., 2016) and Panicum virgatum(Jiang XM et al., 2014). In previous 284 study, our colleague Liang revealed EkGSTU1 performed better than EkGAPDH2 in root, leaf, 285 branch and seed samples (Liang WX et al., 2019). However, in our study, EkGAPDH2 and $286 E k U B C 23$ performed well in different stages of fruit ripening. Our study expanded the scope of 287 reference genes screening for this species. In this study, we selected $E k G A P D H 2$ as the reference 288 gene to quantify the high expression target genes for further study. We also comfirmed $289 E k G A P D H 2$ and $E k U B C 23$ had stable expression in other tissues by semi-quantitative RT-PCR 290 experiment. The RT-PCR experimental method was the same as described above and the details 291 of result were show in Figure S7. The result showed that EkGAPDH2 and EkUBC23 had stable 292 expression in root, branch and leaf, it will facilitate to reference genes screening for whole plant. 293 The genomic information of E. konishii still unclear. The fruit of Euscaphis konishii has high 294 medicinal value, which was used as medicinal source material in China. In order to analyze the 295 expression patterns of medicinal compound related gene in fruit of Euscaphis konishii, we 296 selected two reliable refenrece genes for qRT-PCR normalization. The results will contribute to 297 future studies of the gene expression in E. konishii and genetic studies related to fruit 298 developmental stages. 
300

301

302

303

304

305

306

307

308

309

310

311

312

313

314

315

316

317

318

319

320

321

322

323

324

325

326

327

328

329

330

331

332

333

334

\section{Conclusions}

The development of high-throughput sequencing technology provides an accurate and efficient approach to molecular biology. It plays an important role in molecular breeding and specific gene research. In this study, we construct a screening system for reference genes of E. konishii based on an RNA-seq database from different developmental pericarp samples. We selected 3 stable reference genes ( $E k U B C 23, E k C Y P 38$, and $E k G A P D H 2)$ from 8 candidate reference genes. Among them, $E k U B C 23$ and $E k C Y P 38$ with low expression are suitable for quantifying low expression target genes. However, $E k G A P D H 2$ with a high expression is favourable for quantifying high expression genes. Our study will contribute to future studies of gene expression in E. konishii and genetic studies related to fruit. The results also provided reference for neighboring species.

\section{Acknowledgements}

We are particularly appreciated to Xiaoxing Zou, Zeming Chen, and other members in our research group for their kind suggestions to perfect the experiment.

\section{References}

Andersen CL, Jensen JL, Ørntoft TF, 2004. Normalization of Real-Time Quantitative Reverse Transcription-PCR Data: A Model-Based Variance Estimation Approach to Identify Genes Suited for Normalization, Applied to Bladder and Colon Cancer Data Sets Cancer Res 64(15): 5245-50.

Bustin SA, 2002. Quantification of mRNA using real-time reverse transcription PCR (RT-PCR): trends and problems. Journal of Molecular Endocrinology 29(1): 23-39.

Cheng JJ, Zhang LJ, Cheng HL, Chiou CT, Lee IJ, Kuo YH, 2010. Cytotoxic hexacyclic triterpene acids from Euscaphis japonica. Journal of Natural Products 73(10): 1655-8. Coker J S \& Davies E, 2004. Selection of candidate housekeeping controls in tomato plants using EST data. Biotechniques 35(4): 740-749.

Czechowski T, Stitt M, Altmann T, Udvardi MK, Scheible WR, 2005 Genome-wide identification and testing of superior reference genes for transcript normalization in Arabidopsis. Plant Physiology 139(1): 5-17.

Derveaux S, Vandesompele J \& Hellemans J, 2010. How to do successful gene expression analysis using real-time PCR. Methods 50(4): 227-230.

Dheda K, Huggett JF, Bustin SA, Johnson MA, Rook G, Zumia A, 2004. Validation of housekeeping genes for normalizing RNA expression in real-time PCR. Biotechniques 37, 112 $114,116,118-119$. 
335 Dussert S, Guerin C, Andersson M, Joët T, Tanbarger TJ, Pizot M, Sarah G, Omore A, Durand 336 T,Morcillo F, 2013. Comparative transcriptome analysis of three oil palm fruit and seed tissues 337 that differ in oil content and fatty acid composition. Plant Physiol. 162, 1337-1358.

338 Guo XJ, Chen LN, Yang HQ, 2018. Reference Gene Selection for Quantitative Real-Time PCR 339 in Studying Culm Shape Development of Dendrocalamus sinicus Forest Research 31(02): 120340125.

341 Grabherr MG, Haas BJ, Yassour M, Levin JZ, Thompson DA, Amit I, Adiconis X, Fan L,

342 Raychowdhury R, Zeng QD, Chen ZH, Mauceli E, Hacohen N, Gnirke A, Rhind N, Palma FD,

343 Birren BW, Nusbaum C, Toh KL, Friedman N, Regev A. 2011. Full length transcriptome

344 assembly from RNA seq data without a reference genome. Nature Biotechnology Italic 29: 644345652.

346 Huang Y, Xiang DB, Hu QM, Tan Y, Meng YC, Fei G, 2014. Phenolic acids from fruits of 347 Euscaphis fukienensis Chinese Traditional and Herbal Drugs 45(18): 2611-2613.

348 Huang W, Ding H, Chen LY, Ni L, Ruan YF, Zou XX, Ye M, Zou SQ, 2019. Protective Effect of the Total Triterpenes of Euscaphis konishii Hayata Pericarp on Bacillus Calmette-Guérin Plus Lipopolysaccharide-Induced Liver Injury. Evidence-Based Complementary and Alternative Medicine Article ID 1806021, 15 pages. Jiang XM, Zhang XQ, Yan HD, Zhang Y, Yang ST, Huang LK, 2014. Reference Gene Selection for Real-time Quantitative PCR Normalization in Switchgrass(Panicum virgatum L.) Root Tissue Journal of Agricultural Biotechnology 22(1): 55-63.

355 Kimmy A Stanton, Patrick P Edger, Joshua R Puzey, 2017. A whole-transcriptome approach to evaluating reference genes for quantitative gene expression studies: a case study in mimulus $G 3$

357 (Bethesda) 7(4): 1085-1095.

Kozera B, Rapacz M,2013. Reference genes in Real- time PCR Journal of Applied Genetics

359 2013, 54(4): 391-406.

360 Kumar G \& Singh A K, 2015. Reference gene validation for qRT-PCR based gene expression studies in different developmental stages and under biotic stress in apple Scientia Horticulturae 362 197: 597-606. Liang WX, Ni L, Zou XX, Huang W, Zou SQ, 2018. Research progress on chemical constituents of Euscaphis and their pharmacological effect Chinese Traditional and Herbal Drugs (5): 12201226.

366 Liang WX, Ni L, Rebeca CL, Zou XX, Sun WH, Wu LJ, Yuan XY, Mao, YL, Huang W, Zou 367 SQ, 2019. Comparative transcriptome among Euscaphis konishii Hayata tissues and analysis of 368 genes involved in flavonoid biosynthesis and accumulation BMC genomics 20:24.

369 Liang WX, Zou XX, Rebeca CL, Wu LJ, Sun HW, Yuan XY, Wu SQ, Li PF, Ding H, Ni L, 370 Huang W, Zou SQ, 2018. Selection and evaluation of reference genes for qRT-PCR analysis in 371 Euscaphis konishii hayata based on transcriptome data Plant Methods 14(1): 42.

372 
373 Li QF, Jiang MY, Yu HX, Xin SW, Gu MH, Liu QQ, 2008. Selection of internal reference genes 374 for quantitative RT-PCR analysis of total RNA from endosperm of rice(Oryza sativa L.) Jouranl 375 of YangZhou university (agricultural and life science edition) 29(2): 61- 66.

376 Li YC, Tian K, Sun LJ, Long H, Li LJ, Wu ZZ, 2016. A new hexacyclic triterpene acid from the 377 roots of Euscaphis japonica, and its inhibitory activity on triglyceride accumulation Fitoterapia 378 109: 261-265.

379 Martins PK, Mafra V, Souza WRD, Riberio AP, Vineky F, Basso MF, Cunhua BADB, Molinari $380 \mathrm{BC}, 2016$. Selection of reliable reference genes for RT-qPCR analysis during developmental 381 stages and abiotic stress in setaria viridis Sci Rep 20(6): 28348.

382 Mascia T, Santovito E, Gallitelli D, Fabrizio C, 2010. Evaluation of reference genes for

383

384

385

386

387

388

389

390

391

392

393

394

395

396

397

398

399

400

401

402

403

404

405

406

407

408

409

410

411

412 quantitative reverse transcription polymerase chain reaction normalization in infected tomato plants Molecular Plant Pathology 11(6): 805-816.

Ma SH, Niu HW, Liu CJ, Zhang J, Hou CY, Wang DM, 2013. Expression stabilities of candidate reference genes for rt-qpcr under different stress conditions in soybean. PloS ONE 8(10): e75271. Nolan T, Hands RE \& Bustin SA, 2006. Quantification of mRNA using real-time RT-PCR. Nature Protocols 1(3): 1559.

Pang QQ, Li ZL, Luo SB, Chen RY, Jin KM, Li ZX, Li DM, Sun BJ, Sun GW, 2017. Selection and evaluation of reference genes for qRT-PCR analysis in Eggplant under high temperatures. Horticultural Plant Journal 44(3): 475-486.

Pfaffl MW, Tichopad A, Prgomet C, Neuvians TP, 2004. Determination of stable housekeeping genes, differentially regulated target genes and sample integrity: BestKeeper--Excel-based tool using pair-wise correlations Biotechnology Letters 26(6): 509-515

Sang J, Han XJ, Liu MY, Qiao GR, Jiang J, Zhuo RY, 2013. Selection and validation of reference genes for real-time quantitative PCR in hyper accumulating ecotype of Sedum alfredii under different heavy metals stresses PLOS ONE 8(12): e82927.

Su XJ, Fan BG, Yuan LC, Cui XN, Lu SF, 2013. Selection and Validation of Reference Genes for Quantitative RT-PCR Analysis of Gene Expression in Populus trichocarpa Bulletin of Botany 48 (5): 507-518.

Vandesompele J, Preter K D, Pattyn F, Poppe E, Roy NV, Paepe AD, Speleman F, 2002. Accurate normalization of real-time quantitative RT-PCR data by geometric averaging of multiple internal control genes Genome Biology 3(7): research0034.

Xia W, Mason, Xiao Y, Liu Z, Yang YD, 2014. Analysis of multiple transcriptomes of the African oil palm(Elaeis guineensis) to identify reference genes for RT- qPCR. Journal of Biotechnology 184: 63-73.

Wu JX, Su SY, Fu LL, Zhang YJ, Chai LJ, Yi HL, 2014. Selection of reliable reference genes for gene expression studies using quantitative real-time PCR in navel orange fruit development and pummelo floral organs Scientia Horticulturae 176(2): 180-188.

Wu JY, He B, Du YJ, Li WC, Wei YZ, 2017. Analysis Method of Systematically Evaluating Stability of Reference Genes Using geNorm,NormFinder and BestKeeper XianDai NongYe KeJi ( 5) : 278-281. 
413 Yang D, Li Q, Wang GX,Ma QH, Zhu LQ, 2017. Reference Genes Selection and System

414 Establishment for Real-Time qPCR Analysis in Ping'ou Hybrid Hazelnut Scientia Agricultura

415 Sinica 50(12): 2399-2410.

416 Yeap WC, Loo JM, Wong YC, Kulaveerasingam H, 2014. Evaluation of suitable reference genes

417 for qRT-PCR gene expression normalization in reproductive, vegetative tissues and during fruit

418 development in oil palm Plant Cell Tissue \& Organ Culture 116(1): 55-66.

419 Yuan W, Wan HJ, Yang YJ, 2012. Characterization and Selection of Reference Genes for Real-

420 time Quantitative RT-PCR of Plants Bulletin of Botany 47 (4): 427-436.

421 Yuan XY, Sun WH, Zou XX, Liu BB, Huang W, Chen ZM, Li YL, Qiu MY, Liu ZJ, Mao YL,

422 Zou SQ, 2018. Sequencing of Euscaphis konishii Endocarp Transcriptome Points to Molecular

423 Mechanisms of Endocarp Coloration International Journal of Molecular Sciences 10(19):3209

424 Zhu YY, Wang Y, Zhang H, Li YQ, Guo WD, 2015. Selection and Characterization of Reliable

425 Reference Genes in Chinese Cherry (Prunus pseudocerasus) Using Quantitative Real-time PCR

426 (qRT-PCR) Journal of Agricultural Biotechnology 23(5): 690-700. 
Figure 1

Unigenes lengthdistribution

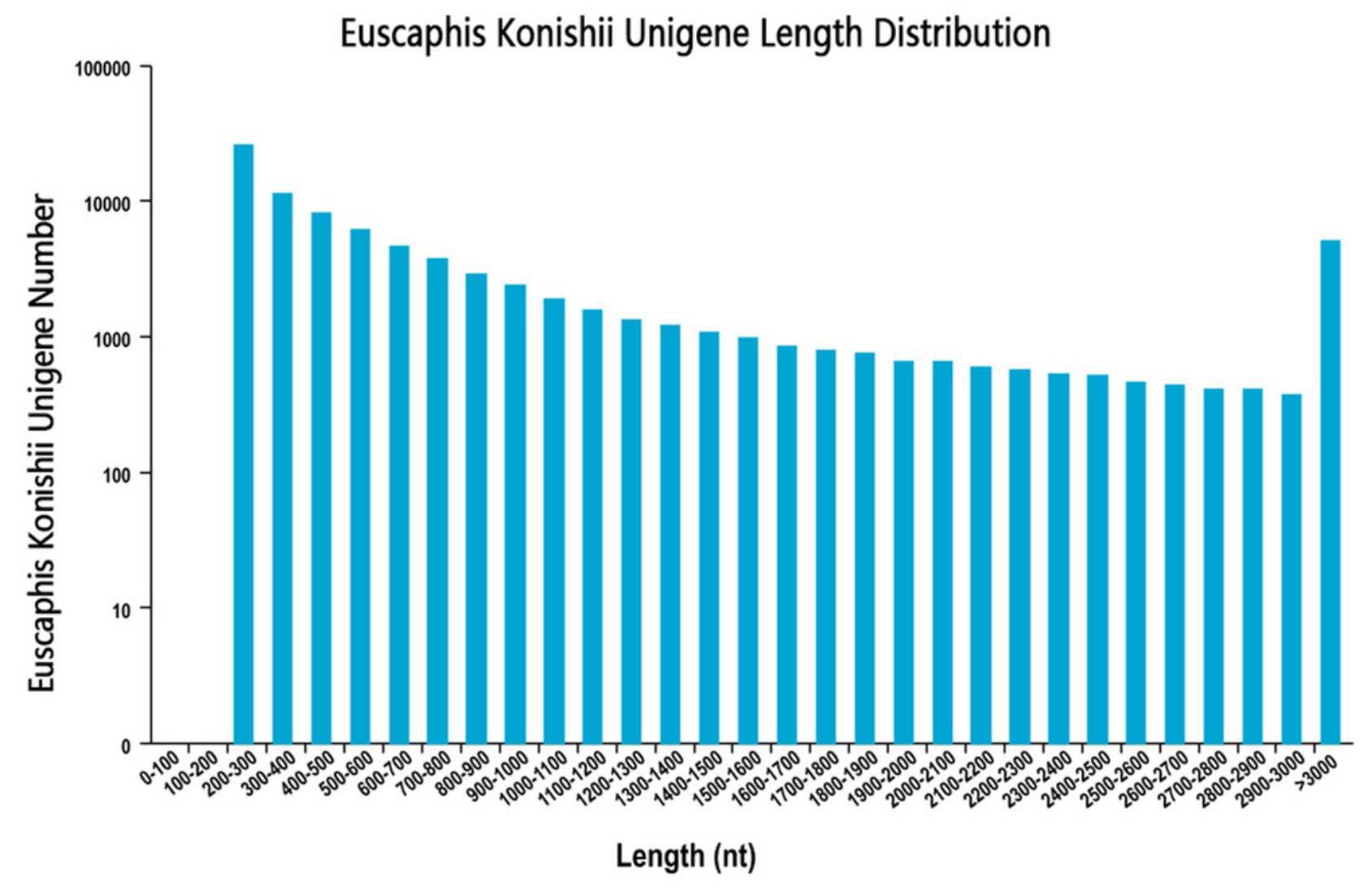


Figure 2

Quantification cycle $(\mathrm{Cq})$ value of eight candidate reference genes across all the experimentalsamples

The green line across the box depicts the median. Whiskers represent the local maximum and minimum values, and points beyond the end of each whisker mark the outliers. The box indicates the $25 \%$ and $75 \%$ percentiles.

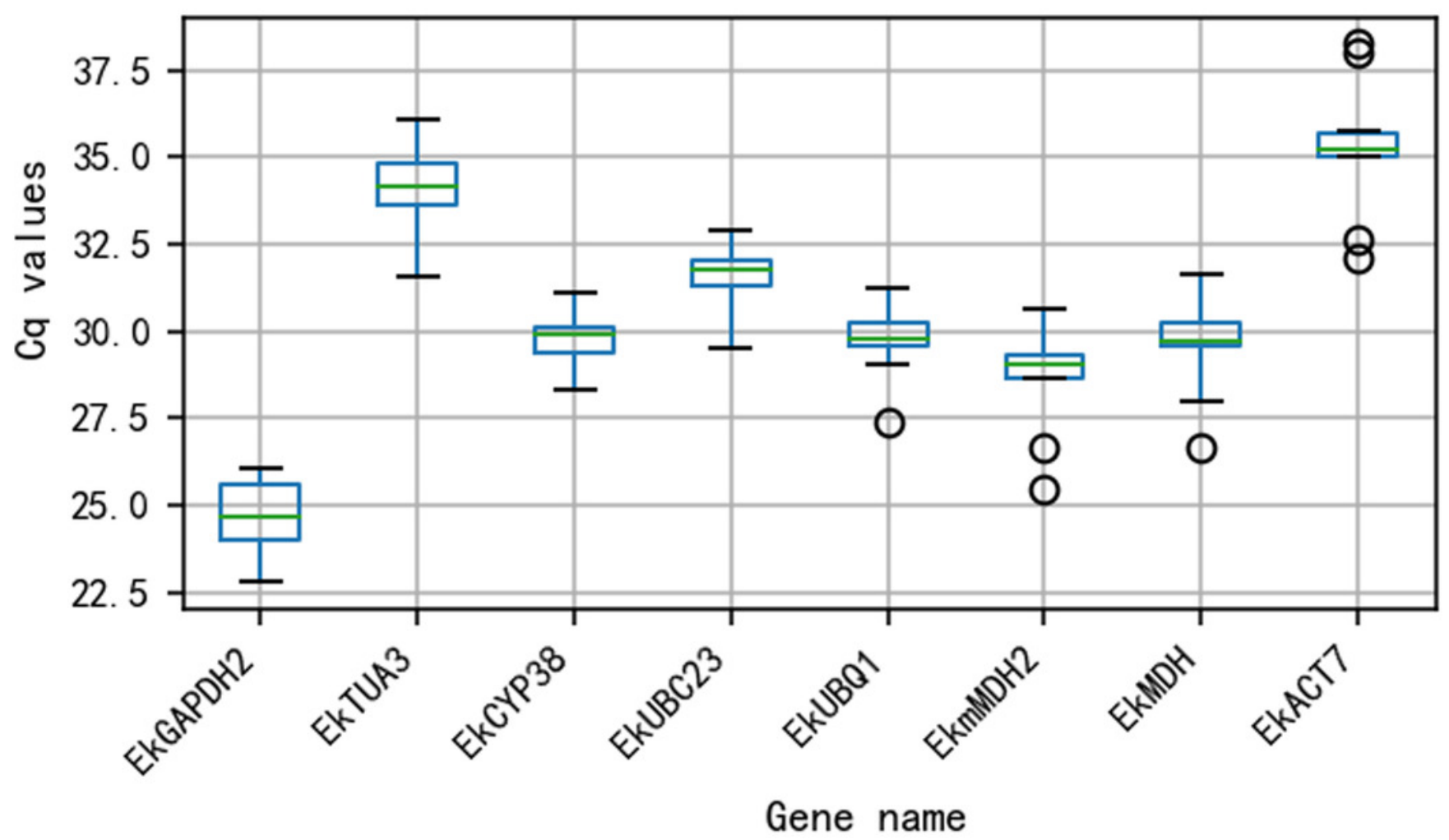




\section{Figure 3}

Expression stablility analysis of reference genes by NormFinder(A), geNorm (B), BestKeeper (C) and ReFinder (D)

Analysis was performed after pooling $\mathrm{Cq}$ data across all three growth conditions. The designation of the analyses were perfomed on all eight candidate reference genes. Gene were ranked from the least stable (on the left) to the most stable (on the right). (A) Genes were ranked according to their NormFinder SV value. (B) Genes were ranked according to their geNorm M value. (C) Genes were ranked according to their BestKeeper SD value. (D) Genes were ranked according to their ReFinder Ranking value.
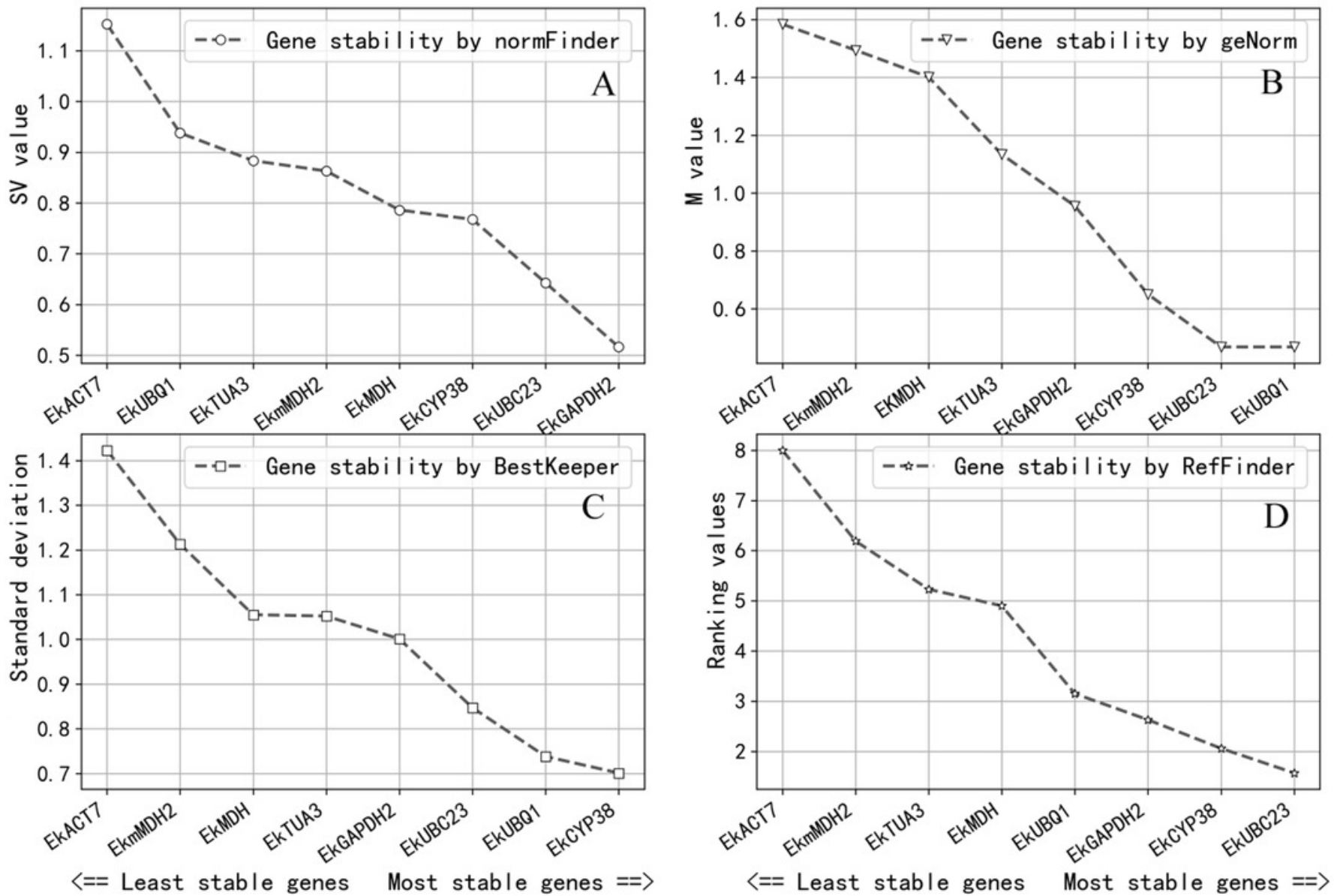
Figure 4

The suitability of reference gene

Figure legend: Genes were normalized to $U B C 23$ and $G A P D H 2$, respectively. Error bars represent standard deviation from theree independent biological replicates. " G", " T" and " $\mathrm{R}^{\prime \prime}$ mean green stage, turing stage and red stage in furit, respectively.

UBC23 $\square$ GAPDH2
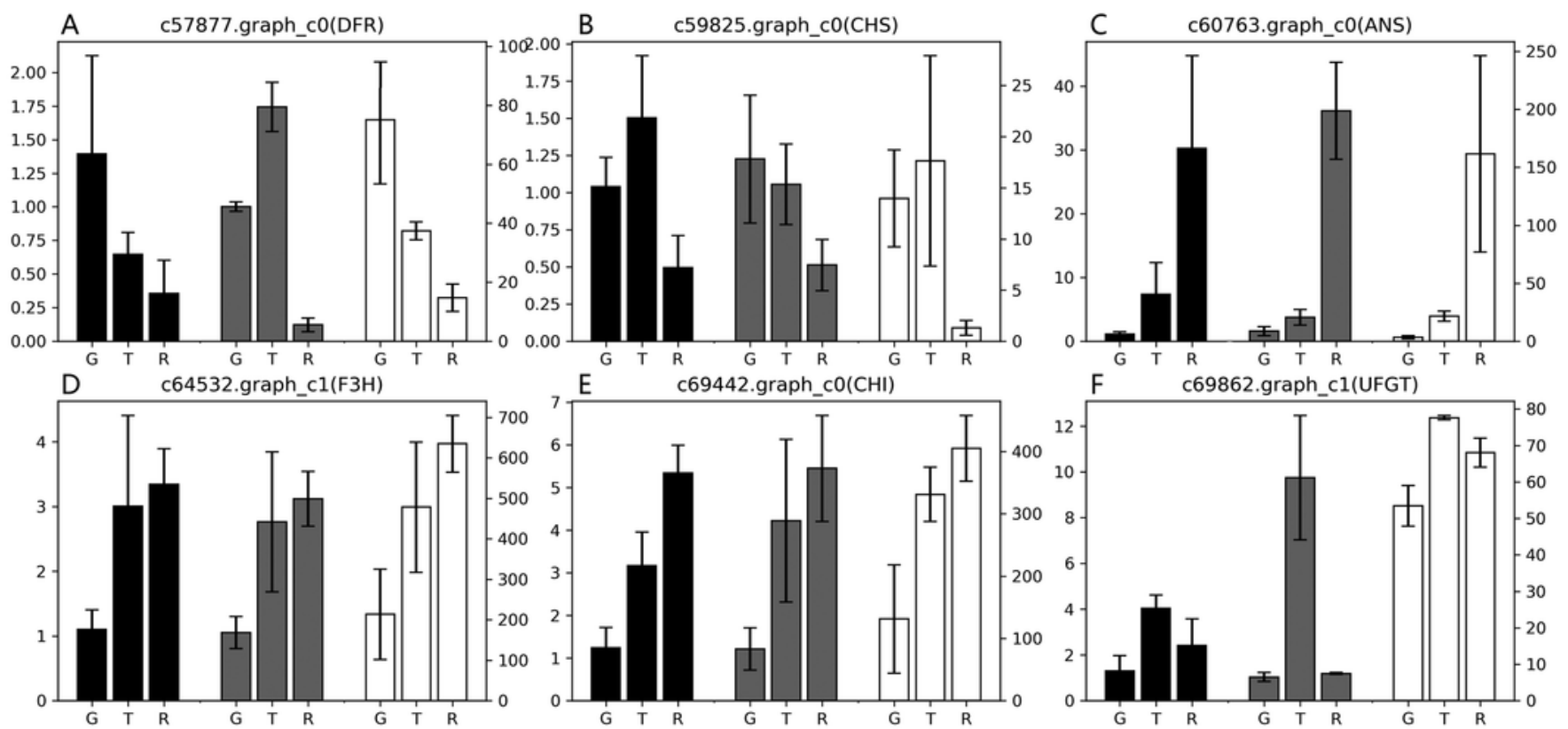

c69442.graph_cO(CHI)
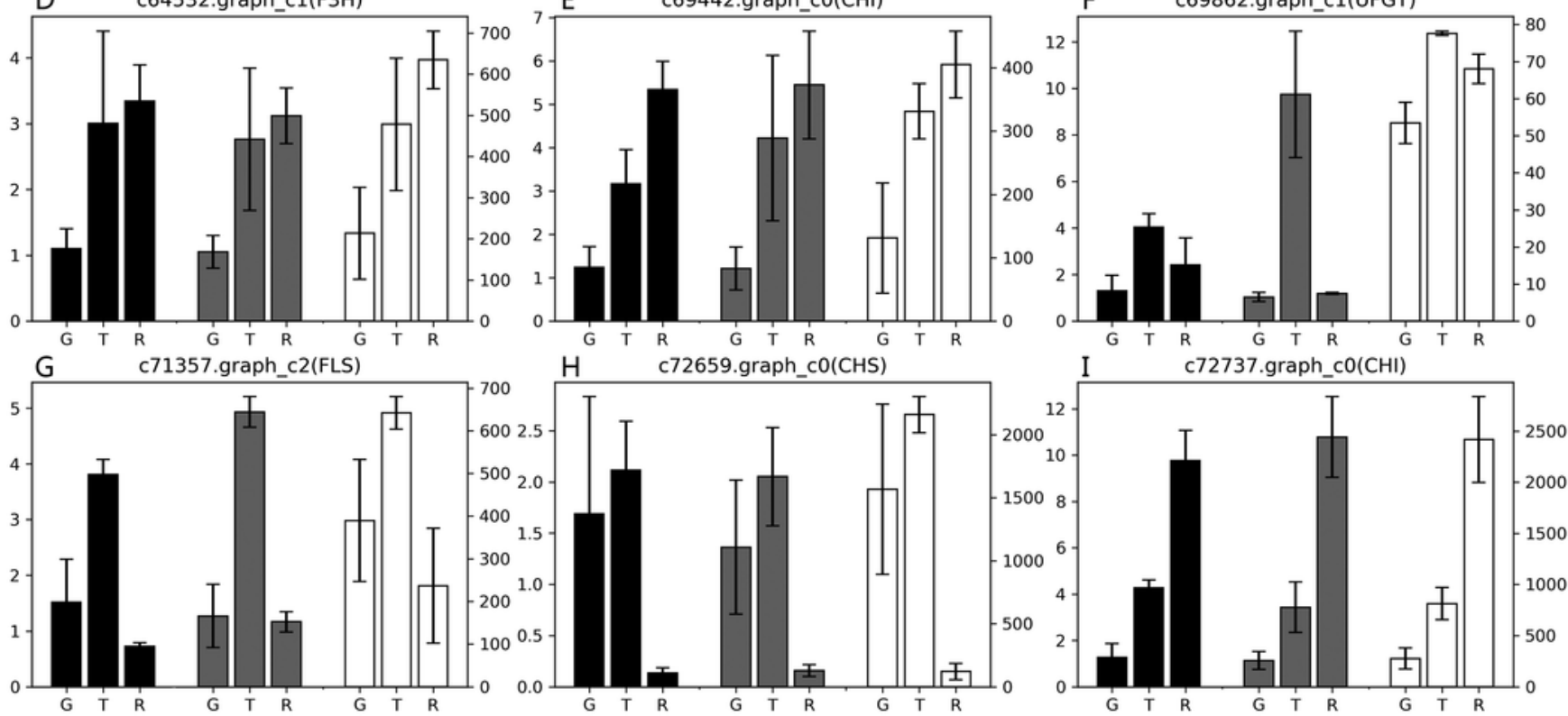


\section{Table $\mathbf{1}$ (on next page)}

Analysis of transcriptomeand expression of candidate reference genes

FPKM, Fragments Per Kilobase of transcript per Million fragments mapped; SD; CV, coefficient variation; FC, flod change; GAPDH2, Glyceraldehyde-3-phosphate dehydrogenase c-2; TUA3, Tubulin alpha-3; CYP38, cyclophilin 38; UBC23, Ubiquitin-conjugating enzyme 23; UBQ1, Ubiquitin extension protein $1 ; m M D H 2$, Lactate/malate dehydrogenase family protein; $M D H$, malate dehydrogenase; $A C T 7$, actin 7. 
1

\begin{tabular}{|c|c|c|c|c|c|c|c|c|c|}
\hline \multirow[b]{2}{*}{ \#ID } & \multirow[b]{2}{*}{$\begin{array}{l}\text { Arabidopsis } \\
\text { ortholog }\end{array}$} & \multirow[b]{2}{*}{ Gene } & \multirow{2}{*}{$\begin{array}{c}\text { E- } \\
\text { value }\end{array}$} & \multirow[b]{2}{*}{$\begin{array}{c}\text { FPKM } \\
\text { Mean }\end{array}$} & \multirow[b]{2}{*}{ SD } & \multirow[b]{2}{*}{$\mathrm{CV}$} & \multicolumn{3}{|c|}{$\mathrm{FC}$} \\
\hline & & & & & & & FC-1 & FC-2 & FC-3 \\
\hline c71660.graph_c1 & AT1G13440.1 & GAPDH2 & $4 e-34$ & 1418.24 & 145.33 & 0.10 & 0.13 & 0.08 & 0.05 \\
\hline c67439.graph_c2 & AT5G19770.1 & $T U A 3$ & $3 e-68$ & 165.32 & 18.28 & 0.11 & 0.12 & 0.05 & 0.08 \\
\hline c67539.graph_c0 & AT3G01480.1 & CYP38 & 1e-59 & 19.10 & 3.07 & 0.16 & 0.08 & 0.08 & 0.01 \\
\hline c67010.graph_c0 & AT2G16920.1 & $U B C 23$ & $2 \mathrm{e}-158$ & 18.30 & 2.05 & 0.11 & 0.19 & 0.13 & 0.04 \\
\hline c62586.graph_c0 & AT3G52590.1 & $U B Q 1$ & $3 e-10$ & 230.36 & 21.98 & 0.10 & 0.19 & 0.00 & 0.18 \\
\hline c65728.graph_c0 & AT3G15020.2 & $m M D H 2$ & $5 e-35$ & 187.30 & 18.88 & 0.10 & 0.11 & 0.20 & 0.08 \\
\hline c63030.graph_c0 & AT3G47520.1 & $M D H$ & $2 \mathrm{e}-19$ & 19.87 & 1.95 & 0.10 & 0.16 & 0.14 & 0.03 \\
\hline c63658.graph_c0 & AT5G09810.1 & $A C T 7$ & $2 \mathrm{e}-38$ & 407.67 & 35.20 & 0.09 & 0.09 & 0.17 & 0.08 \\
\hline
\end{tabular}

2 
Table 2 (on next page)

Genes and primer sets usedfor qRT-PCR analysis

$F$, forward primer; $R$, reverse primer. 
1

\begin{tabular}{|c|c|c|c|c|c|}
\hline \#ID & Gene name & Primer sequence & Length & Distance from $3^{\prime}$ & Anneal Temp \\
\hline \multirow{2}{*}{ c71660.graph_c1 } & \multirow{2}{*}{ GAPDH2 } & F:CCGTGTTCCTACTGTTGATGT & \multirow{2}{*}{95} & 1244 & 62.0 \\
\hline & & R:CCTCCTTGATAGCAGCCTTAAT & & 1338 & 61.9 \\
\hline \multirow{2}{*}{ c67439.graph_c2 } & \multirow{2}{*}{ TUA3 } & F:GGGTGGTAGCAAACCCTATTAC & \multirow{2}{*}{103} & 203 & 62.4 \\
\hline & & R:CCGAAGGTGCAGATGATGAA & & 305 & 62.2 \\
\hline \multirow{2}{*}{ c67539.graph_c0 } & \multirow{2}{*}{ СYР38 } & F:ATCTGTTGGAACTCCTCCATTC & \multirow{2}{*}{114} & 2251 & 62.0 \\
\hline & & R:AGCCCTGAAGCAAGGTAAAG & & 2364 & 62.2 \\
\hline \multirow{2}{*}{ c67010.graph_c0 } & \multirow{2}{*}{$U B C 23$} & F:AGCCACATAATCTCCGTGTAAG & \multirow{2}{*}{105} & 4161 & 62.0 \\
\hline & & R:GCTGACCATGTTCGAGTAGTT & & 4265 & 62.0 \\
\hline \multirow{2}{*}{ c62586.graph_c0 } & \multirow{2}{*}{$U B Q 1$} & F:ACGAGCCAAAGCCATCAA & \multirow{2}{*}{105} & 1435 & 62.0 \\
\hline & & R:GGCCGAACTCTTGCTGATTA & & 1539 & 62.2 \\
\hline \multirow{2}{*}{ c65728.graph_c0 } & \multirow{2}{*}{$m M D H 2$} & F:CATCGTAAGTCCCTGCTTTCT & \multirow{2}{*}{104} & 956 & 61.9 \\
\hline & & R:TGCCAAGTACTGCCCTAATG & & 1059 & 62.0 \\
\hline \multirow{2}{*}{ c63030.graph_c0 } & \multirow{2}{*}{$M D H$} & F:ATGAAGAAGTCCACGAGCTAAC & \multirow{2}{*}{97} & 995 & 62.1 \\
\hline & & R:GCCATAGACAGAGTAGCAGAAC & & 1091 & 62.0 \\
\hline \multirow{2}{*}{ c63658.graph_c0 } & \multirow{2}{*}{$A C T 7$} & F:GATCTGGCATCACACCTTCTAC & \multirow{2}{*}{112} & 394 & 62.3 \\
\hline & & R:CTGAGTCATCTTCTCCCTGTTG & & 505 & 62.0 \\
\hline
\end{tabular}

2 


\section{Table 3(on next page)}

Target genes and primers.

F, forward primer; $R$, reverse primer. 
1

Locus Primer sequence $\left(5^{\prime}-3^{\prime}\right)$

c50541.graph_c0 (CHS) F:GGAACTCGCTGTTCTGGATAG / R:CCTTGTGGCCCTTAACTTCT

c59825.graph_c0 (CHS) F:GCATGTGTTGTGCGAGTATG / R:CCTTCCCTTCTTCCAGAGATTT

c60763.graph_c0 (ANS) F:CAGCTTGAGTGGGAAGACTATT / R:TACTCGCTTGTTGCCTCTATG

c64532.graph_c1 (F3H) F:GGTTCAAGATTGGCGTGAAATAG / R:CATCAGCTTCCCACTGTACTC

c69442.graph_c0 (CHI) F:TCTTGCTGAGGATGATGACTTT / R:TCTCTAGCTGCACTCCATACT

c69862.graph_c1 (UFGT) F:ACCGCTAATCCCAACTCTTTC / R:GTGGTTCGGTGTGCCTATT

c71357.graph_c2 (FLS) F:CGACAATCGCTCCATCTTCT / R:ATGGCCTCCTTCCTGTATTAAC

c72659.graph_c0 (CHS) F:TTGGTGACGCCGAAGATAAA / R:GAGGTCCAGCTACAGTTCTTG

c72737.graph_c0 (CHI) F:CTCTTGTCCAGCAGCATTCTA / R:CAGAGTTTGGCTGCAAGAATATC

c58939.graph_c0(SS3) F: TGAATGGATGCAGGTGACTGGAAC / R: CCACACTTGCTGAGTTGCTCTTTC

2 